\title{
Intestinal perception: mechanisms and assessment
}

\author{
Fernando Azpiroz* \\ Digestive System Research Unit, University Hospital Vall d'Hebron, Autonomous University of Barcelona, Barcelona 08035, Spain
}

\begin{abstract}
Physiological stimuli in the gut induce regulatory reflexes to accomplish the digestive process, but are normally not perceived. However, under some circumstances, gut stimuli may activate perception pathways and induce conscious sensations. Experimental evidence gathered during the past decade suggests that patients with functional gut disorders and unexplained abdominal symptoms may have a sensory dysfunction of the gut, so that physiological stimuli would induce symptoms. Assessment of visceral sensitivity is still poorly developed, but in analogy to somatosensory testing, differential stimulation of visceral afferents may be achieved by a combination of stimulation techniques, which may help to characterize sensory dysfunctions. Visceral afferent input is modulated by a series of mechanisms at different levels of the brain-gut axis, and conceivably, a dysfunction of these regulatory mechanisms could cause hyperalgesia. The sensory dysfunction in functional patients seems to be associated with altered reflex activity, and both mechanisms may interact to produce the symptoms. Evidence of a gut sensory-reflex dysfunction as a common pathophysiological mechanism in different functional gastrointestinal disorders would suggest that they are different forms of the same process, and that the clinical manifestations depend on the specific pathways affected.
\end{abstract}

Visceral sensitivity: Visceral afferents: Gut reflexes: Functional gut disorders

\section{Clinical importance of gut perception}

The gut is a profusely innervated organ able to sense and react to a variety of stimuli. Indeed, the digestive function is accomplished by a complex feedback regulation that involves a net of reflexes (Fig. 1). During ingestion, the stomach relaxes to accommodate the meal and initiates the gastric phase of digestion (Moragas et al. 1993). A progressive gastric contraction during the postprandial period produces gastric emptying at a rate perfectly adapted to the processing capability of the small intestine. Likewise, intestinal motility and transit are metered to achieve optimal absorption of nutrients, and this process is regulated by a series of intestinointestinal reflexes depending on the composition of chyme along the small bowel. These regulatory mechanisms adapt the digestive function to a wide variety of situations, and under normal conditions the whole process is not perceived. However, a dysfunction of the digestive system may result in the perception of abdominal symptoms, and indeed this is a very common condition in clinical practice: more than half of the patients in a gastroenterological clinic complain of abdominal symptoms, without any organic cause demonstrable by conventional diagnostic tests. In the absence of positive diagnostic findings, these conditions have been categorized as functional gastrointestinal disorders, or more precisely, disorders of gastrointestinal function.

Among this broad and heterogeneous category of disorders, several syndromes, such as non-cardiac chest pain, functional dyspepsia and irritable bowel syndrome (IBS), have been defined based on the clinical symptoms (Thompson et al. 1992). Non-cardiac chest pain refers to patients with thoracic symptoms without cardiac, pulmonary or oesophageal diseases. Functional dyspepsia applies to symptoms such as epigastric pressure, fullness and bloating, that presumably originate from the upper gastrointestinal tract, and that are frequently precipitated by meals. IBS is attributable to the distal gut, and is characterized by abdominal pain or discomfort associated with disordered bowel habit. The diagnosis of these syndromes is based on clinical criteria, because their underlying pathophysiology is unknown.

Some reports in the 1970s described disturbances of gut perception in patients with the irritable bowel and related syndromes, but these studies remained largely ignored. These classic observations were later reconfirmed and expanded, clearly showing a colonic and rectal hypersensitivity in these patients (Whitehead et al. 1990; Mayer \& Gebhart, 1994; Distrutti et al. 2004). Further studies revealed the symptoms after meal ingestion in patients with functional dyspepsia were due to a sort of gastric rigidity, that is, to

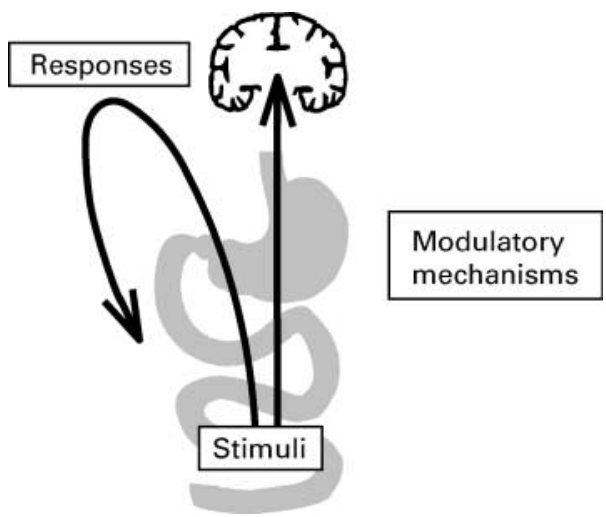

Fig. 1. Responses to gut stimuli: gut stimuli normally induce reflexes that regulate the digestive function. However, under some circumstances, gut stimuli may activate perception afferents and induce symptoms. Both reflexes and perception are modulated by a variety of mechanisms at 
altered compliance and an abnormal response of the stomach to distension. Gastric accommodation to a meal was experimentally reproduced by distending the stomach with an air-filled bag, either with fixed volumes or at fixed pressure levels maintained by a barostat. With both methods the results were equivalent. Gastric distension studies showed that the pressure-volume relationship, that is, compliance, was normal. However, the patients developed their customary symptoms at distending levels that were largely unperceived by healthy subjects (Mearin et al. 1991). It is important to note that these studies were performed in fasted subjects, and hence, did not entirely reproduce the conditions of meal accommodation. Nevertheless, these data suggested that dyspeptic symptoms could be related to gastric hypersensitivity. Similar types of gut hypersensitive responses were also recognized in patients with non-cardiac chest pain (Mayer \& Gebhart, 1994), and these data altogether suggested that patients with functional gut disorders could have a sensory dysfunction, so that physiological stimuli induced symptoms. These studies attracted much attention to the field of visceral perception that was so far poorly investigated. The methodology used for somatosensory testing was applied to study gastrointestinal perception and new specific tools have been developed.

Further studies have characterized the sensory dysfunctions in patients with functional gut syndromes. In the first place, it has been well established that the dysfunction affects exclusively the visceral territory. It has been shown that somatic sensitivity, both to the cold pressure test and to transcutaneous electrical nerve stimulation, is normal in dyspeptic patients (Mearin et al. 1991; Coffin et al. 1994a). Furthermore, the visceral responses to somatic pain are also normal in these patients. Specifically, somatic pain produced by cold stress induces a gastric relaxation, which is similar in dyspeptic patients and in healthy subjects (Mearin et al. 1991). IBS patients have been also shown to display increased tolerance of somatic pain (Accarino et al. 1995), and this attenuated somatosensory response has been related to the pain-reporting behaviour characteristic of painful conditions.

Several studies have attempted to define the regions of the gut and the specific pathways affected in different subsets of patients, but still the extension of the gut sensory dysfunction in patients with functional disorders remains controversial. Increased gastric but normal duodenal sensitivity was shown in a specific subset of dyspeptic patients predominantly complaining of postcibal bloating (Coffin et al. 1994a; Caldarella et al. 2003). In this study dyspeptic patients invariably recognized that gastric distension, but not duodenal distension, reproduced their customary symptoms, whereas in healthy subjects both stimuli were perceived alike. However, other studies have reported increased perception of intestinal distension in patients with functional dyspepsia (Greydanus et al. 1991). Patients with functional dyspepsia are heterogeneous and the criteria for definition and selection of the patients may explain the conflicting data. In IBS patients colonic hypersensitivity to distension has been well documented (Whitehead et al. 1990; Mayer \& Gebhart, 1994), but it seems that more proximal portions of the gut, such as the jejunum and even the oesophagus, are also affected (Moriarty \& Dawson, 1982; Accarino et al. 1995).

\section{Methods to evaluate gut perception}

Assessment of visceral sensitivity requires probing stimuli that activate afferent pathways and induce perception. The peripheral neuron of this viscero-sensory system follows sympatheticsplanchnic pathways up to the spinal cord (Fig. 2). Sensory endings in the gut wall can be experimentally stimulated by gut distension. Distension of the gastrointestinal tract can be performed by means of a distending device, a balloon or a similar device, mounted over a tube. High-compliance latex balloons made with condoms have relatively low intrinsic pressure, and compliance can be calculated with a reasonably small error. Flaccid bags with negligible intrinsic pressure require no corrections and may be preferable. However, the bag has to be oversized, because when the capacity of the bag is attained during a distension, the gut is not being really tested.

Distensions can be simply produced by manual inflation using a syringe or with more sophisticated methods. For instance, the barostat applies any desired intraluminal pressure level by a feedback regulation of the volume of air within an intraluminal bag (Azpiroz \& Malagelada, 1987; Notivol et al. 1995). The tensostat is a similar feedback device that applies fixed tension levels to the gut wall by means of a computerized air pump (Distrutti et al. 1999, 2004; Caldarella et al. 2003). Recent studies have shown that perception of gut distension relies on stimulation of tension receptors, while intraluminal volume and wall stretch seem not to be determinant (Distrutti et al. 1999, 2004). Hence, the tensostat probably allows a better standardization of distending stimuli, regardless of the capacity and compliance of the organ tested.

Gastrointestinal distension induces symptoms, such as abdominal pressure and fullness, referred to the epigastrium and the paraumbilical region, which are similar to those reported by patients with functional gastrointestinal disorders. The symptomatic response to distension is rather homogeneous from the stomach down to the mid small bowel (Azpiroz \& Malagelada, 1990; Rouillon et al. 1991; Accarino et al. 1992, 1995; Distrutti et al. 2004), which indicates that both the expression of the gut in response to stimuli and the discriminative value of symptoms in relation to the site of origin in the gut are relatively poor. A small proportion of distensions in the stomach and proximal duodenum induces nausea, which is rarely induced by jejunal distension. In contrast, jejunal distensions are frequently perceived as colicky or stingy sensations.

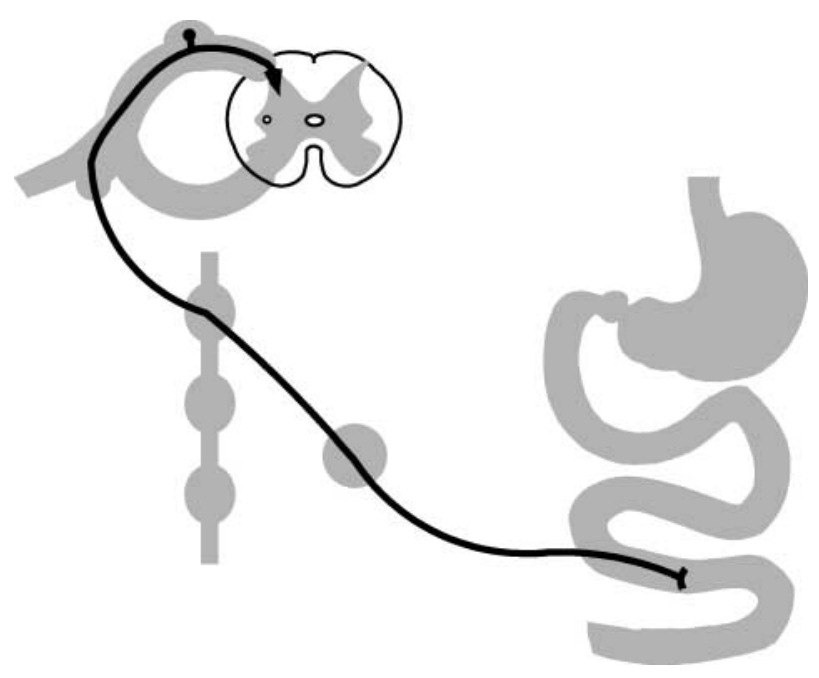

Fig. 2. Intestinal perception afferents. The peripheral neuron of the viscerosensory system follows sympathetic-splanchnic pathways up to the spinal cord. 
Perception of the gut motility can be evaluated by detection of sensory thresholds using various paradigms of stimuli presentation. The intensity and the quality of perception can be measured by means of rating scales, which may be analogue, numerical or descriptive. The intensity of perception is stimulus-related, but the same types of sensation are induced by distensions from the threshold for perception up to the threshold for discomfort. Furthermore, the sensitivity along the gastrointestinal tract seems quite uniform, although standardization of distending stimuli in different segments of the gut has been problematic. There is little experience about the affective (unpleasant) dimension of visceral sensation, which seems independent of the intensity of perception.

New imaging techniques, such as positron emission tomography, single-photon emission computer tomography and functional magnetic resonance imaging, use different tracers to detect focal changes in brain blood flow and metabolic activity in response to different stimuli. These techniques provide images of the brain regions activated by visceral stimulation, but their application is limited by their restricted availability.

In analogy to somatosensory testing, which involves a variety of stimulation techniques to activate specific pathways, alternative stimuli to distension have been tested in the gut. Transmucosal electrical nerve stimulation has been applied in the gut via intraluminal electrodes mounted over a tube (Accarino et al. 1992, 1995). Hence, whereas distending stimuli activate sensory pathways and induce perception by specific stimulation of mechanoreceptors on the gut wall, transmucosal nerve stimulation induces similar perception by non-specific stimulation of afferent pathways, that is, without relying on any specific receptor. Studies using both mechanical stimuli and transmucosal nerve stimulation have shown that patients with IBS have hypersensitivity of the small bowel, which selectively affects mechanosensitive afferents, with normal perception of electrical stimulation (Accarino et al. 1995). Methods for thermal stimulation, involving both cold and warm stimuli, have also been developed to test visceral afferents (Villanova et al. 1997). Thermal stimulation of the gut can be produced via intraluminal bags by recirculating water at adjusted temperatures. It has been shown that the stomach and the intestine exhibit similar stimulus-related thermal sensitivity, but still gastrointestinal thermal sensitivity in man and specifically the type of afferents activated by warm and cold stimuli remain poorly explored. Nevertheless, thermal stimuli are potentially applicable in conjunction with mechanical and electrical stimuli for the evaluation of gastrointestinal sensory dysfunctions.

\section{Modulation of gut perception}

Perception of gut stimuli is modulated by a variety of mechanisms at different levels between the gut and the brain. In the first place, the responses to gut stimuli depend on both the intensity of stimulation and the number of receptors activated. It has been shown that the summation phenomena may substantially modify gut perception in man (Serra et al. 1995). Specifically, the surface of intestine exposed to a distending stimulus determines the intensity of perception. Furthermore, summation effects are similar regardless of whether adjacent or distant fields are stimulated, at least over the proximal half of the small bowel. These observations suggest that the intestine may tolerate circumscribed activation of sensory terminals without perception, but recruitment of additional areas of the gut, even at distant sites, may induce symptoms. This reasoning may apply to a variety of pathological conditions, but it may be particularly relevant for the interpretation of a common clinical problem, such as symptoms induced by intestinal gas distension. These data would explain why a focal gas collection may be unperceived, whereas pooling of intestinal gas may be symptomatic, even without inordinate intraluminal pressures.

Visceral perception is also modified by the interaction of different stimuli in the gut (Accarino et al. 2001, 2002). For instance, intestinal lipids increase the sensitivity of the intestine to mechanical stimuli, and this effect is not attributable to changes in the intestinal compliance. However, the sensitization induced by lipids seems specifically related to mechanoreceptors. Indeed, intraluminal lipids do not modify perception of transmucosal electrical stimulation of the gut (Accarino et al. 2001). Cholecystokinin could play a role in these effects, because it has been shown to sensitize gut mechanoreceptors. Furthermore, in the presence of intestinal lipids, loxiglumide, a cholecystokinin type A receptor antagonist, reduces perception of gastric distension.

In a series of studies in the UK it was shown that patients with IBS and functional abdominal pain have a distorted referral pattern of gut sensations and perceive intestinal distensions over the abdomen more diffusely than healthy controls (Moriarty \& Dawson, 1982). Visceral and somatic afferents converge onto the same sensory neurons in the spinal cord, and sensitization of these neurons by noxious visceral input produces an expansion of their somatic receptive fields (Mayer \& Gebhart, 1994). Peripheral hypersensitivity of mechanosensitive pathways could produce a secondary sensitization of spinal neurons, which could explain the expanded referral area of gut stimuli in IBS (Mayer \& Gebhart, 1994).

A complex neural circuitry, which can be activated by somatic stimuli, is involved in the modulation of pain perception. It seems that a neuronal link at the brain stem exerts a descending inhibitory control over spinal transmission, as well as at higher levels of the somatic projection system. Conceivably, spinal and supraspinal circuits with specific modulatory effects can be activated depending on the type of stimulation. This modulatory system also controls visceral sensitivity. Specifically, it has been shown that the application of transcutaneous electrical nerve stimulation on the hand reduces the discomfort produced by gastric and duodenal distensions. This form of viscero-sensory modulation by somatic afferents is exerted without alteration of basal gut tone or visceral reflexes (Coffin et al. 1994b). Some forms of counter irritation require painful stimulation, but visceral discomfort can be reduced by painless somatic stimuli. Furthermore, somatic stimuli may decrease perception of uncomfortable, but not necessarily painful, visceral sensations. These observations in man are supported by experimental studies showing that somatic and visceral sensory input converge onto the same spinal neurons, and that these somatovisceral neurons can be modulated by both segmental and descending inhibitory mechanisms (Ness \& Gebhart, 1990). The pathophysiological role of these mechanisms in functional gut disorders remains speculative, but impairment of such a down-regulation mechanism could be implicated in the pathogenesis of the visceral hypersensitivity. Furthermore, therapeutic techniques to induce visceral hypoalgesia through somatic stimulation could potentially benefit patients with abdominal symptoms. It seems that acupuncture operates by the same type of pain control mechanisms as those activated by other forms 
of somatic stimulation, and hence, may have similar effects (Melzack, 1994).

The autonomic nervous system may also induce visceral sensory dysfunctions. Specifically, increased sympathetic tone magnifies perception of gut stimuli, without affecting reflex responses (Iovino et al. 1995). Alteration of the sympathetic modulation of visceral sensitivity may be clinically relevant. Recent studies have shown that patients with IBS display increased sympathetic activity (Aggarwal et al. 1994), and precisely these patients exhibit a similar sensory disturbance to that produced by sympathetic activity, namely, they manifest visceral hypersensitivity, but normal or even increased tolerance to somatic stimuli (Whitehead et al. 1990; Accarino et al. 1995).

Visceral perception is finally determined at a cortical level, and hence, may be influenced by cognitive mechanisms. For instance, it has been shown that anticipatory knowledge increases the perception of intestinal distension as compared to mental distraction (Accarino et al. 1997). Furthermore, anticipatory knowledge increases the referral area, and the stimuli were perceived over a wider abdominal region. Mental activity does not modify intestinal compliance or the reflex response to distension. Hence, cognitive processes selectively regulate the sensitivity to gut stimuli, while visceral reflexes operate independently. These data raise the possibility that functional patients may pay more attention to gut events. Psychological mechanisms also modulate gut perception. It has been shown in healthy subjects that symptoms of colonic distension are modulated by anxiety induced by mental stress and, to a lesser intent, by active relaxation (Ford et al. 1995). Hypnotherapy, which may activate this type of cognitive and psychological mechanisms, has been shown to reduce perception of rectal distension in some patients with IBS and improve their clinical outcome (Whorwell, 1991).

\section{Correlation of perception and gut reflexes}

Gut stimuli may also reduce reflex motor responses. Indeed, motility of the digestive tract is normally regulated by a series of reflexes, involving both vagal and sympathetic pathways. Reflex responses to gut stimuli in man can be investigated in the laboratory using different methods to measure gut motor activity. The gut generates both phasic-pulse and tonic-sustained contractions. Phasic activity can be recorded by measuring pressure changes within the gut using conventional manometry. Tonic contractions do not produce detectable changes in intraluminal pressure, and thus, evaluation of tonic activity requires a more sophisticated methodology. Changes in the gut tone can be measured by means of the barostat as changes in the volume of air within an intraluminal bag, maintained at a fixed pressure level by an electronic air pump. When the gut relaxes the barostat injects air to prevent a pressure fall, and when the gut contracts the barostat withdraws air. Using this isobaric approach, a volume expansion reflects a relaxation, and a volume reduction a contraction (Azpiroz \& Malagelada, 1987). The barostat has proven particularly useful for studying reflex activity, because brief inhibitory reflexes may be missed by recording intermittent phasic activity (Azpiroz \& Malagelada, 1990; Rouillon et al. 1991).

In contrast to the uniformity of perception, the reflex responses to gut distension are quite heterogeneous, and some data indicate that perception and reflex responses are dissociable and probably mediated by different mechanisms (Azpiroz \& Malagelada, 1990; Rouillon et al. 1991). From a pathophysiological standpoint this finding may be very important because it means that perception and reflex responses to gastrointestinal stimuli may be independently altered in some conditions. Indeed, some data indicate that despite the fact that gross motor abnormalities cannot be detected in patients with functional gut disorders using conventional techniques, more refined studies on reflex activity indicate that the dysfunction in these patients involves not only sensory pathways, but regulatory motor pathways as well. For instance, it has been shown that dyspeptic patients with gastric hypersensitivity have also impaired gastric reflexes (Coffin et al. 1994a; Caldarella et al. 2003). Normally, duodenal balloon distension induces a gastric relaxation, and this enterogastric reflex is driven by the vagus. In a group of dyspeptic patients with normal duodenal sensitivity and compliance, duodenal distension induced impaired relaxation of the stomach. Further studies have shown that physiological reflexes are also affected. Normally intestinal nutrients induce a gastric relaxation, which is a feedback mechanism to control nutrient outflow from the stomach. It has been shown that these types of reflexes are also impaired in patients with functional dyspepsia. Impaired vagal function could explain the defective duodenogastric reflex (Greydanus et al. 1991). The intestinal motor responses to distension, that is, the sympathetic intestino-intestinal reflexes, may also be impaired in dyspepsia (Greydanus et al. 1991).

Ingestion of a meal induces a relaxation of the proximal stomach to accommodate the meal without increments in intragastric pressure (Moragas et al. 1993). Nutrient-dependent enterogastic reflexes regulate the magnitude of the relaxation, and, as the relaxatory input decreases, the proximal stomach gently forces the intragastric content into the antrum. A gastric hyporeactivity to enterogastric reflexes would predictably result in a defective accommodation of the proximal stomach and an antral overload. Reduced fundal residency and increased antral filling has been reported in patients with functional dyspepsia (Troncon et al. 1994). Impaired meal accommodation, in turn, would potentiate hypersensitivity to distension, because the stomach tolerates smaller volumes when not properly relaxed (Notivol et al. 1995). Furthermore, some experimental data indicate that increased intragastric pressure after a meal, simulating a defective gastric accommodation, produces dyspeptic-type symptoms without disturbing gastric emptying (Moragas et al. 1993), a condition that resembles most patients with functional dyspepsia (Tucci et al. 1992). Hence, it is plausible that the impaired gastric reflex exacerbates the poor tolerance of dyspeptics to intragastric volumes, and thus, contributes to the generation of clinical symptoms in the absence of major motor dysfunctions.

Studies on IBS patients have demonstrated that rectal hypersensitivity is associated with motor hyperactivity in response to gut stimuli (Whitehead et al. 1990). Again both hypersensitivity and hyperreactivity could contribute to the perception of rectal tenesmus and faecal urgency, which is a common symptom in these patients. Recent studies using a new methodology to evaluate intestinal gas dynamics further substantiate the role of combined sensory-reflex disturbances. These studies have shown that gas symptoms in IBS patients may be related to impaired intestinal handling of gas leading to retention, gut hypersensitivity with poor gas tolerance or both (Serra et al. 2001, 2002).

The concomitant dysfunction of sensory and reflex pathways has not been satisfactorily explained. This association could be due to a process that affects the gut wall, or it may be alternatively explained on the basis of a multifocal or diffuse 
gut neuropathy, but confirmatory evidence is still lacking. Nevertheless, evidence of gut sensory-reflex dysfunctions as a common pathophysiological mechanism in various functional gut syndromes would suggest that they are different forms of the same process. Altered reflex activity and altered conscious perception of gut stimuli may combine in different degrees, and their interaction may explain the generation of clinical symptoms. Based on this disease model, symptoms in patients with functional gut disorders, which are heterogeneous and frequently overlapping, may depend on the neurological mechanisms and the territories affected.

\section{Conclusion and implications}

Firm evidence collected over the past few years indicates that visceral sensitivity disturbances may be implicated in patients with functional disorders of the gut. Furthermore, sensory disturbances seem to be associated with altered gut reflexes and these mixed sensory-reflex dysfunctions may explain the development of clinical symptoms. However, the putative causes and the clinical implications of these disturbances still remain to be established. These findings prompted much research in the field of gastrointestinal perception, as well as the development of specific methodologies for testing sensations elicited by gut stimuli. The concept of unspecific gastrointestinal well-being has been proposed, but experimental evidence will have to be produced (Benton \& Donohoe, 1999; Cummings et al. 2001; Smith et al. 2001). The existence of specific pleasant sensations derived from the gastrointestinal tract is supported by uncontrolled observations. Such sensations are primarily related to the intake of meals and the evacuation of faeces - in particular, gratifying sensations such as satiation and complete rectal evacuation - and conceivably also preparatory sensations, such as appetite or call for stools. Other physiological events, such as eructation and flatus, and non-specific sensations, such as 'easy digestion', may also contribute to gastrointestinal well-being. In contrast to perception of symptoms ('ill-being'), very little is known about gastrointestinal 'well-being' and perception of pleasant sensations originating in the gut. However, the conceptual and methodological developments derived from pathophysiological studies could be applied for this purpose.

\section{References}

Accarino AM, Azpiroz F \& Malagelada J-R (1992) Symptomatic responses to stimulation of sensory pathways in the jejunum. Am J Physiol 263, G673-G677.

Accarino AM, Azpiroz F \& Malagelada J-R (1995) Selective dysfunction of mechanosensitive intestinal afferents in the irritable bowel syndrome. Gastroenterology 108, 636-643.

Accarino AM, Azpiroz F \& Malagelada J-R (1997) Attention and distraction: effects on gut perception. Gastroenterology 113, 415-422.

Accarino AM, Azpiroz F \& Malagelada J-R (2001) Modification of small bowel mechanosensitivity by intestinal fat. Gut 48, 690-695.

Accarino AM, Azpiroz F \& Malagelada J-R (2002) Gut perception in humans is modulated by interacting gut stimuli. Am J Physiol 282, G220-G225.

Aggarwal A, Cutts TF, Abell TL, Cardoso S, Familoni B, Bremer J \& Karas J (1994) Predominant symptoms in irritable bowel syndrome correlate with specific autonomic nervous system abnormalities. Gastroenterology 106, 945-950.
Azpiroz F \& Malagelada J-R (1987) Gastric tone measured by an electronic barostat in health and postsurgical gastroparesis. Gastroenterology 92, 934-943.

Azpiroz F \& Malagelada J-R (1990) Perception and reflex relaxation of the stomach in response to gut distention. Gastroenterology 98, 1193-1198.

Benton D \& Donohoe RT (1999) The effects of nutrients on mood. Public Health Nutr 2, 403-409.

Caldarella P, Azpiroz F \& Malagelada J-R (2003) Antro-fundic dysfunctions in functional dyspepsia. Gastroenterology 124, 1220-1229.

Coffin B, Azpiroz F, Guarner F \& Malagelada J-R (1994a) Selective gastric hypersensitivity and reflex hyporeactivity in functional dyspepsia. Gastroenterology 107, 1345-1351.

Coffin B, Azpiroz F \& Malagelada J-R (1994b) Somatic stimulation reduces perception of gut distension. Gastroenterology 107, 1636-1642.

Cummings JH, Christie S \& Cole TJ (2001) A study of fructo oligosaccharides in the prevention of travellers' diarrhoea. Aliment Pharmacol Ther 15, 1139-1145.

Distrutti E, Azpiroz F, Soldevilla A \& Malagelada J-R (1999) Gastric wall tension determines perception of gastric distension. Gastroenterology 116, $1035-1042$.

Distrutti E, Salvioli B, Azpiroz F \& Malagelada J-R (2004) Rectal function and bowel habit in irritable bowel syndrome. Am J Gastroenterol 99, $131-137$.

Ford MJ, Camilleri M, Zinsmeister AR \& Hanson RB (1995) Psychosensory modulation of colonic sensation in the human transverse and sigmoid colon. Gastroenterology 109, 1772-1780.

Greydanus MP, Vassallo M, Camilleri M, Nelson DK, Hanson RB \& Thomforde GM (1991) Neurohormonal factors in functional dyspepsia: insights on pathophysiological mechanisms. Gastroenterology 100, 1311-1318.

Iovino P, Azpiroz F, Domingo E \& Malagelada J-R (1995) The sympathetic nervous system modulates perception and reflex responses to gut distension in humans. Gastroenterology 108, 680-686.

Mayer EA \& Gebhart GF (1994) Basic and clinical aspects of visceral hyperalgesia. Gastroenterology 107, 271-293.

Mearin F, Cucala M, Azpiroz F \& Malagelada J-R (1991) The origin of symptoms on the brain gut axis in functional dyspepsia. Gastroenterology 101, 999-1006.

Melzack R (1994) Folk medicine and the sensory modulation of pain. In Textbook of Pain, 3rd ed., pp. 1209-1217 [PD Wall and R Melzack, editors]. Edinburgh: Churchill Livingstone.

Moragas G, Azpiroz F, Pavía J \& Malagelada J-R (1993) Relations among intragastric pressure, postcibal perception and gastric emptying. Am J Physiol 264, G1112-G1117.

Moriarty KJ \& Dawson AM (1982) Functional abdominal pain: further evidence that the whole gut is affected. Br Med $J$ 284, 1670-1672.

Ness TJ \& Gebhart GF (1990) Visceral pain: a review of experimental studies. Pain 41, 167-386.

Notivol R, Coffin B, Azpiroz F, Mearin F, Serra J \& Malagelada J-R (1995) Gastric tone determines the sensitivity of the stomach to distension. Gastroenterology 108, 330-336.

Rouillon JM, Azpiroz F \& Malagelada J-R (1991) Sensorial and intestinointestinal reflex pathways in the human jejunum. Gastroenterology 101, 1606-1612.

Serra J, Azpiroz F \& Malagelada J-R (1995) Perception and reflex responses to intestinal distension are modified by simultaneous or previous stimulation. Gastroenterology 109, 1742-1749.

Serra J, Azpiroz F \& Malagelada J-R (2001) Impaired transit of intestinal gas in the irritable bowel syndrome. Gut 48, 14-19.

Serra J, Salvioli B, Azpiroz F \& Malagelada J-R (2002) Lipid-induced intestinal gas retention in the irritable bowel syndrome. Gastroenterology 123, 700-706.

Smith A, Bazzoni C, Beale J, Elliot-Smith J \& Tiley M (2001) High fibre breakfast cereals reduce fatigue. Appetite 37, 1-3.

Thompson WG, Creed F, Drossman DA, Heaton KW \& Mazzacca G (1992) Functional bowel disease and functional abdominal pain. Gastroenterol Int 5, 75-91. 
Troncon LEA, Bennett RJM, Ahluwalia DK \& Thompson DG (1994) Abnormal intragastric distribution of food during gastric emptying in functional dyspepsia patients. Gut 35, 327-332.

Tucci A, Corinaldesi R, Stanghellini V, et al. (1992) Helicobacter pylori infection and gastric function in patients with chronic idiopathic dyspepsia. Gastroenterology 103, 768-774.

Villanova N, Azpiroz F \& Malagelada J-R (1997) Perception and gut reflexes induced by stimulation of gastrointestinal thermoreceptors in humans. J Physiol (Lond) 502, 215-222.

Whitehead WE, Holtkotter B, Enck P, Hoelzl R, Holmes KD, Anthony J, Shabsin HS \& Schuster MM (1990) Tolerance for rectosigmoid distension in irritable bowel syndrome. Gastroenterology 98, 1187-1192.

Whorwell PJ (1991) Use of hypnotherapy in gastrointestinal disease. $\mathrm{Br} \mathrm{J}$ Hosp Med 45, 27-29. 